\title{
RBM24 exacerbates bladder cancer progression by forming a Runx1t1/TCF4/miR-625-5p feedback loop
}

Yue-Wei Yin ${ }^{1,2}$, Kai-Long Liu ${ }^{1,2}$, Bao-Sai Lu ${ }^{1,2}$, Wei Li ${ }^{1,2}$, Ya-Lin Niu' ${ }^{1}$ Chen-Ming Zhao ${ }^{1}$, Zhan Yang ${ }^{1,2}$, Ping-Ying Guo ${ }^{1,2}$ and Jin-Chun Qi ${ }^{1,2}$

\begin{abstract}
RNA-binding motif protein 24 (RBM24) acts as a multifunctional determinant of cell fate, proliferation, apoptosis, and differentiation during development by regulating premRNA splicing and mRNA stability. It is also implicated in carcinogenesis, but the functions of RBM24 in bladder cancer (BC) remain unclear. In the present study, we revealed that RBM24 was upregulated in BC tissues. Importantly, we found that a higher level of RBM24 was correlated with poor prognosis in BC patients. Overexpression of RBM24 promoted BC cell proliferation, while depletion of RBM24 inhibited BC cell proliferation in vivo and in vitro. Mechanistically, RBM24 positively regulated Runx1t1 expression in BC cells by binding to and enhancing Runx1t1 mRNA stability. Furthermore, Runx1t1 in turn promoted RBM24 expression by interacting with the transcription factor TCF4 and suppressing the transcription of miR-625-5p, which directly targets RBM24 and suppresses RBM24 expression. RBM24-regulated BC cell proliferation was moderated via the Runx1t1/TCF4/miR-625-5p feedback loop. These results indicate that the RBM24/Runx1t1/TCF4/miR-625-5p positive feedback loop participates in BC progression. Disruption of this pathway may be a potential therapeutic strategy for BC treatment.
\end{abstract}

\section{Introduction}

Bladder cancer $(\mathrm{BC})$ is one of the most common malignancies in the urinary system, with an estimated 450,000 new cases per year worldwide ${ }^{1}$. Among these cases, approximately $70 \%$ of new cases are nonmuscleinvasive $\mathrm{BC}$ (NMIBC), while $30 \%$ are muscle-invasive $\mathrm{BC}$ $(\mathrm{MIBC})^{2}$. Surgery, chemotherapy, and radiation therapy are the main treatments for $\mathrm{BC}$ patients ${ }^{3}$. However, the 5 -year survival rate of high-risk patients is still very low, and the current main treatment methods cannot prevent the recurrence or progression of these $\mathrm{BC}$ patients ${ }^{4}$. One important reason is the poor understanding of the mechanisms underlying $\mathrm{BC}$ development and progression.

\footnotetext{
Correspondence: Ping-Ying Guo (guopingying1@sina.com) or Jin-Chun Qi (hburology2017s@163.com)

'Department of Urology, The Second Hospital of Hebei Medical University, Shijiazhuang 050000, P.R. China

${ }^{2}$ Hebei Institute of Urology, Shijiazhuang 050000, P.R. China
}

Therefore, it is necessary to better understand the molecular basis of $\mathrm{BC}$ and explore innovative therapeutic strategies.

RNA-binding motif protein 24 (RBM24) is a multifunctional protein involved in the regulation of premRNA splicing, mRNA stability, and translation, and through these functions, it acts as a critical determinant of cell fate and differentiation ${ }^{5}$. It was once believed that RBM24 is preferentially expressed in cardiac and skeletal muscle tissues and primarily serves to regulate embryonic heart development ${ }^{6,7}$. However, recent studies have demonstrated that RBM24 also regulates cancer progression ${ }^{8}$. Hua et al. found that RBM24 inhibited the progression of nasopharyngeal carcinoma by upregulating miR-25, which in turn downregulated MALAT1 ${ }^{9}$. Using immunoprecipitation coupled to reverse transcription and microarray analysis (RIP-ChIP), Yu et al. demonstrated that RBM24 is a multitasking RNA-binding protein (RBP) capable of

\section{(c) The Author(s) 2021}

(c) (i) Open Access This article is licensed under a Creative Commons Attribution 4.0 International License, which permits use, sharing, adaptation, distribution and reproduction cc) in any medium or format, as long as you give appropriate credit to the original author(s) and the source, provide a link to the Creative Commons license, and indicate if changes were made. The images or other third party material in this article are included in the article's Creative Commons license, unless indicated otherwise in a credit line to the material. If material is not included in the article's Creative Commons license and your intended use is not permitted by statutory regulation or exceeds the permitted use, you will need to obtain permission directly from the copyright holder. To view a copy of this license, visit http://creativecommons.org/licenses/by/4.0/. 
regulating the stability and expression of multiple bound targets ${ }^{10}$. RBPs may function as suppressors or facilitators of disease depending on the specific upstream regulators and downstream effectors (targets) ${ }^{3}$. However, the role of RBM24 in BC is still unclear.

Runt-related transcription factor 1 (Runx1t1) is a member of the eight-twenty-one (ETO) family of proteins ${ }^{11}$. Runx1t1 was first identified through its involvement in a $t$ $(8 ; 21)$ translocation associated with acute myeloid leukemia $(\mathrm{AML})^{12}$. Subsequent studies reported that Runx1t1 acts as a transcriptional corepressor by interacting with DNAbound transcription factors and recruiting other proteins to facilitate transcriptional repression ${ }^{13}$. A recent study found upregulated expression of Runx1t1 in cord blood-derived endothelial colony-forming cells ${ }^{14}$. In accord with the function of Runx1t1 in vascular endothelial development, a Runx1t1-deficient mouse showed reduced angiogenesis ${ }^{15}$. However, Runx1t1 was also reported to suppress colorectal cancer by regulating cell proliferation and chemotherapeutic drug resistance ${ }^{16}$. Runx1t1 may upregulate the levels of the cell cycle genes Cdk4 and Cdk6 by recruiting a histone deacetylase (HDAC)-containing nuclear corepressor complex ${ }^{17,18}$. However, the role of Runx1t1 in BC remains unclear.

In the present study, we found that higher RBM24 and Runx1t1 levels in $B C$ tissue were correlated with poor patient survival in $\mathrm{BC}$ patients. Moreover, overexpression of RBM24 upregulated Runx1t1 by stabilizing Runx1t1 mRNA and concomitantly accelerated BC proliferation. We identified a protein-protein interaction between Runx1t1 and TCF4 that suppressed miR-625-5p at the transcriptional level, which acted as a negative regulator of RBM24 by directly targeting RBM24. Taken together, our results indicate that RBM24, Runx1t1, TCF4, and miR-625-5p form a positive feedback loop that can drive the proliferation of BC cells. The RBM24/Runx1t1/TCF4/ miR-625-5p pathway may be a potential therapeutic target for $\mathrm{BC}$ treatment.

\section{Materials and methods}

\section{Clinical samples}

Human primary BC tissues and the corresponding normal bladder tissues were collected from $\mathrm{BC}$ patients who were admitted to the Department of Urology of the Second Hospital of Hebei Medical University from July 2015 to June 2019. All BC patients were histopathologically and clinically diagnosed and were treated with radical cystectomy. The study protocol was approved by the Ethics Committee of Second Hospital of Hebei Medical University, and written consent was obtained from each patient.

\section{Cell lines and transfection}

The normal uroepithelial cell SV-HUC-1 was purchased from the Cell Bank of the Chinese Academy of Sciences, and bladder cancer cell lines UM-UC-3, 253 J, T24, and J82 were purchased from ATCC (Rockville, Maryland). The 293 A cell line was collected in our lab. All cells were cultured in DMEM supplemented with $10 \%$ fetal bovine serum (FBS) and 1\% penicillin/streptomycin. Cells were grown in a humidified atmosphere of $95 \%$ air and $5 \% \mathrm{CO}_{2}$. Transfection was performed by using Lipofectamine 2000 (Invitrogen) according to the manufacturer's protocols. The miR-625-5p, miR-149-3p, and miR-449a mimic, mimic NC, miR-625-5p inhibitor, inhibitor NC, shRBM24, shRunx1t1, shTCF4, and negative controls were purchased from GenePharma Co., Ltd. (Shanghai, China). The oeRBM24 and oeRunx1t1 overexpression plasmids were obtained from GENEWIZ Company (Suzhou, China).

\section{RNA extraction and real-time quantitative PCR}

Clinical tumor tissues and xenograft tissues were lysed using QIAzol Lysis Reagent (79306). Total RNA was isolated with a miRNeasy Mini Kit (217004; Qiagen) according to the manufacturer's protocols. A NanoDrop 2000 was used to determine RNA quality. For miRNA, the miScript II RT Kit (218161) and miScript SYBR Green PCR Kit (Catalog No. 218073) were used to perform reverse transcription and quantitative real-time (qRT)PCR according to the manufacturer's protocols with primers listed in Table 1. For mRNA, cDNA was synthesized using an M-MLV First Strand Kit (Life Technologies) with random hexamer primers. mRNAs were subjected to qRT-PCR using the Platinum SYBR Green qPCR SuperMix UDG Kit (Invitrogen) and the ABI 7500 FAST System (Life Technologies) with primers listed in Table 1. The relative transcript expression levels were normalized to GAPDH and calculated using the $2^{-\Delta \Delta C t}$ formula as previously described ${ }^{19}$.

\section{Biotin-labeled RNA synthesis and pull-down}

Biotin-labeled RNA was synthesized via in vitro transcription as previously described ${ }^{3}$. The RBM24 $3^{\prime}$ UTR containing T7 promoter sequences was amplified with or without Biotin-16-UTP (Ambion, AM8452) by using the MEGAscript T7 Transcription Kit (Ambion, AM1334). The miRNeasy Mini Kit (217004; Qiagen) was used to purify the transcribed RNA, and biotin pull-down was carried out to detect interactions between the RBM24 $3^{\prime}$ UTR and microRNAs as previously described ${ }^{19}$. In brief, cells were transfected with $4 \mu \mathrm{g}$ of biotin-labeled RNA for $24 \mathrm{~h}$. Then, the cells were cross-linked with $1 \%$ formaldehyde in PBS and quenched with $0.125 \mathrm{M}$ glycine. The cells were resuspended in lysis buffer on ice for $10 \mathrm{~min}$ and sonicated. The cell lysate was diluted two times with hybridization buffer. Streptavidin Dynabeads (Life Technologies) were blocked for $2 \mathrm{~h}$ at $4{ }^{\circ} \mathrm{C}$ in lysis buffer containing $1 \mathrm{mg} / \mathrm{ml}$ yeast tRNA and $1 \mathrm{mg} / \mathrm{ml} \mathrm{BSA}$ and washed twice with $1 \mathrm{ml}$ lysis buffer. Then, $100 \mu \mathrm{l}$ of 
Table 1 Oligos used in this study.

\begin{tabular}{|c|c|c|c|}
\hline Oligo name & Sequence $\left(5^{\prime}-3^{\prime}\right)$ & Purpose & Amplicon (bp) \\
\hline RBM24-F & GCTGGATGCCGGTTGTTAAG & RT-qPCR for RBM24 mRNA. & 357 \\
\hline RBM24-R & GCACAAAAGCCTGCGGATAG & & \\
\hline TCF4-F & AGCAGAGTCTCCTTGGAGGT & RT-qPCR for TCF4 mRNA. & 206 \\
\hline TCF4-R & AGTGCTTGCTGATGGAGCAT & & \\
\hline RUNX1T1-F & TCACACACAATGTGCCATCCT & RT-PCR for RUNX1T1 mRNA. & 273 \\
\hline RUNX1T1-R & TCGGTGAGTCCTGTCTGGAT & & \\
\hline GAPDH-F & AAGGTGAAGGTCGGAGTCAAC & RT-qPCR for GAPDH & 102 \\
\hline GAPDH-R & GGGGTCATTGATGGCAACAATA & & \\
\hline miR-29a-F & GGACTGATTTCTITGGTGTTCAG & RT-qPCR for miR-29a & N/A \\
\hline miR-138-3p-F & GCTACTTCACAACACCAGGGC & RT-qPCR for miR-138-3p & N/A \\
\hline miR-149-3p-F & AGGGAGGGACGGGGGCTG & RT-qPCR for miR-149-3p & N/A \\
\hline miR-216a-5p-F & GGTAATCTCAGCTGGCAACTGTG & RT-qPCR for miR-216a-5 & $\mathrm{N} / \mathrm{A}$ \\
\hline miR-222-5p-F & GGCCTCAGTAGCCAGTGTAGATC & RT-qPCR for miR-222-5p & $\mathrm{N} / \mathrm{A}$ \\
\hline miR-625-5p-F & GGCAGGGGGAAAGTTCTATAGTCC & RT-qPCR for miR-625-5p & N/A \\
\hline miR-449a-F & GGTGGCAGTGTATTGTTAGCTGG & RT-qPCR for miR-449a & N/A \\
\hline miR-578-F & GGCCTTCTTGTGCTCTAGGATTG & RT-qPCR for miR-578 & N/A \\
\hline miR-1273-F & CGGGCGACAAAGCAAGACTC & RT-qPCR for miR-1273 & N/A \\
\hline RNU6-1(U6)-F & GTGCTCGCTTCGGCAGCACATATAC & RT-qPCR for the internal control U6 & 106 \\
\hline RNU6-1(U6)-R & AAAATATGGAACGCTTCACGAATTTGC & & \\
\hline miR-625-ChIP-1-F & TGGCTCCGCCCCCTITCAG & ChIP-PCR for miR-625-5p promoter & 176 \\
\hline miR-625-ChIP-1-R & GACTGCTGAGCCTGCCACTCC & & \\
\hline miR-625-ChIP-2-F & GAAGTGGCAGCGGGAACAGG & ChIP-PCR for miR-625-5p promoter & 225 \\
\hline miR-625-ChIP-2-R & CCAGGAGCAGGCAGCAGCC & & \\
\hline miR-625-ChIP-3-F & CCCAGGAGCCTGTCTGCTTCC & ChIP-PCR for miR-625-5p promoter & 167 \\
\hline miR-625-ChIP-3-R & GGATATCATCACAGCCCAACAGG & & \\
\hline RBM24-3'UTR-F & CTCGAGACCAGCCATCTGATCAAAGTTG & PCR amplification of the RBM24 $3^{\prime}$ UTR for cloning & 1756 \\
\hline RBM24-3'UTR-R & GAATTCGAAGTITTAAAAATTATATTTAATAC & & \\
\hline RBM24-3'UTR-T7-F & $\begin{array}{l}\text { GGATCCTAATACGACTCACTATAGGACCAG } \\
\text { CCATCTGATCAAAGTTG }\end{array}$ & $\begin{array}{l}\text { PCR amplification of the RBM24 } 3^{\prime} U T R \\
\text { sequence for transcription in vitro }\end{array}$ & 1756 \\
\hline RBM24-3'UTR-T7-R & GAATTCGAAGTITTTAAAAATTATATTTAATAC & & \\
\hline
\end{tabular}

the washed/blocked Dynabeads was added to the lysate, and the mixture was rotated for $30 \mathrm{~min}$ at $37^{\circ} \mathrm{C}$. The beads were captured by magnets (Life Technologies) and washed five times. The beads were then subjected to RNA elution with buffer.

\section{Coimmunoprecipitation (ColP) assay}

Three micrograms of antibodies and protein A-agarose were added to $253 \mathrm{~J}$ cell lysates for $12 \mathrm{~h}$ at $4{ }^{\circ} \mathrm{C}$. Then, protein A-agarose-antigen-antibody complexes were collected by centrifugation. After washing with IP buffer and extensive washing with lysis buffer, the immunoprecipitates were resolved by $10 \%$ SDS-PAGE, followed by western blot analysis.

\section{Proximity ligation assay}

The proximity ligation assay (PLA) was performed as described previously ${ }^{19}$. Briefly, $253 \mathrm{~J}$ cells were seeded into six-well chamber slides and cultured for $24 \mathrm{~h}$. Then, $4 \%$ paraformaldehyde was used to fix the slides. Anti-Runx1t1 and anti-TCF4 antibodies were used to stain the slides. Rabbit PLUS and Mouse MINUS Duolink In Situ 
Proximity Ligation Assay (PLA) kits were used to detect interactions between the two proteins following the manufacturer's protocols. Fluorescence was detected using a laser scanning confocal microscope.

\section{Western blot analysis}

Western blot analysis was carried out as described previously $^{20}$. Protein was extracted from the cultured cells and frozen tissue samples with RIPA lysis buffer. Equal amounts of protein were run on SDS-PAGE and electrotransferred to polyvinylidene fluoride (PVDF) membranes (Millipore) that were then blocked with $5 \%$ milk for $2 \mathrm{~h}$. Then, the membranes were incubated with primary antibodies overnight at $4{ }^{\circ} \mathrm{C}$. The antibodies that were used were as follows: antiRBM24 (1:500, 18178-1-AP), anti-STAT3 (1:1000, 10253-2AP), anti-CDK4 (1:1000, 60186-1-Ig), anti-IGF1R (1:500, 20254-1-AP), anti-Runx1t1 (1:1000, 15494-1-AP), antiEGLN1 (1:500, 66589-1-Ig), anti-TCF4 (1:500,22337-1-AP), and anti- $\beta$-actin (1:1000, sc-47778). The membranes were then incubated with the HRP-conjugated secondary antibody (1:5000, Rockland) for $1 \mathrm{~h}$ at room temperature. The blots were treated with ImmobiloTM Western (Millipore) and detected by ECL (enhanced chemiluminescence) Fuazon Fx (Vilber Lourmat). Images were captured and processed by FusionCapt Advance Fx5 software (Vilber Lourmat). All experiments were replicated three times.

\section{Vector construction and luciferase reporter assay}

The $3^{\prime}$ untranslated region (UTR) sequence of RBM24 containing the wild-type form of the miR-625-5p target site was inserted into the Xho1- and EcoR1-digested psiCHECK vector (Promega Corp.). The 2-kb miR-625 promoter sequence was obtained by PCR with primers and inserted into the Mlu1- and Xho1-digested pGL3-basic vector (Promega Corp., Madison, WI, USA). A luciferase assay was performed as described previously ${ }^{21}$. In brief, $293 \mathrm{~A}$ cells were seeded into a 24-well plate and cotransfected with the RBM24 reporter construct (wild-type or mutant) or the empty reporter vector and either miR-625-5p mimic and pRL-TK or mimic control and pRL-TK; additionally, $293 \mathrm{~A}$ cells were cotransfected with the pGL3-miR-luc vector or oeRBM24 or shTCF4 for $24 \mathrm{~h}$. Luciferase activity was measured by the Dual-Glo Luciferase Assay System (Promega, Madison, WI) with a Flash and Glow (LB955, Berthold Technologies) reader. The specific target activity was expressed as the relative ratio of firefly luciferase activity to Renilla luciferase activity.

\section{Xenograft animal model}

The xenograft model was generated as described previously ${ }^{3,20,22}$. Male BALB/c nude mice at 4-6 weeks of age (18-22 g) were purchased from Vital River Laboratory Animal Technology Co., Ltd. (Beijing, China). A total of $5 \times 10^{6}$ stable shRBM24/shRunx1t1-infected $253 \mathrm{~J}$ cells were harvested via trypsinization and resuspended in $0.2 \mathrm{~mL}$ PBS mixed with 50\% Matrigel (Collaborative Research Inc., Bedford, MA, USA); this suspension was injected subcutaneously into the right dorsal flank. The length and width of mouse tumors were measured twice a week with a calipers. Then, we used the following formula to calculate the tumor volume: tumor volume $=$ (length $\times$ width $\left.^{2}\right) / 2$. At the end of this experiment, the mice were euthanized by carbon dioxide asphyxiation. All animal experiments were approved by the Institutional Animal Care and Use Committee of Hebei Medical University (Approval ID: HebMU 20080026) and were designed to minimize suffering.

\section{Morphometry and histology}

Human bladder cancer and normal bladder tissues were fixed with formalin solution and then processed for routine embedding in paraffin. Ten consecutive $5-\mu \mathrm{m}$-thick sections were prepared for hematoxylin and eosin staining. Cross-section images were acquired using a Leica microscope (Leica DM6000B, Switzerland) and digitized with LAS V.4.4 (Leica).

\section{Immunofluorescence staining}

Five-micrometer paraffin-embedded cross-sections of tissues were subjected to immunofluorescence staining as described previously ${ }^{22}$. Sections were deparaffinized with xylene, rehydrated, and preincubated with $10 \%$ normal goat serum (710027, KPL, USA) followed by incubation with the following primary antibodies: anti-RBM24 (181781-AP) and anti-Runx1t1 (67086-1-Ig). The sections were subsequently treated with the following secondary antibodies: fluorescein-labeled anti-rabbit IgG (021516; KPL, USA) and rhodamine-labeled anti-mouse IgG (031806; KPL). In each experiment, DAPI (157574; MB Biomedical) was used for nuclear counterstaining. Images were captured using a confocal microscope (DM6000 CFS; Leica) and processed using LAS AF software.

\section{MTT assay}

Cell viability was detected by MTT [3-(4,5-dimethylthiazol-2-yl)-2,5-diphenyltetrazolium bromide] colorimetric assay. Briefly, $253 \mathrm{~J}$ and $\mathrm{J} 82$ cells were plated in 96well plates and treated with actinomycin (Act) for 0, 2, 4, and $8 \mathrm{~h}$. Then, $20 \mu \mathrm{L}$ of MTT reagent $(5 \mathrm{mg} / \mathrm{mL}$; SigmaAldrich) was added to each well and incubated for 3-4 h, and the absorbance was measured at $570 \mathrm{~nm}$ using a microplate reader (Thermo Fisher, USA).

\section{RNA immunoprecipitation (RIP) assays}

$253 \mathrm{~J}$ cells were transfected with pWPI or oeRBM24 for $48 \mathrm{~h}$, and then cells were used to conduct RIP experiments using an RBM24 antibody (18178-1-AP) or IgG and the Dynabeads $^{\mathrm{TM}}$ Protein G Immunoprecipitation Kit 
(10007D, Thermo Fisher) according to the manufacturer's instructions. The RNA fraction isolated by RIP was quantified by a NanoDrop 2000 (Thermo-Fisher). cDNA was synthesized using an M-MLV First Strand Kit (Life Technologies) with random hexamer primers. The STAT3, GAPDH, and Runx1t1 RIP primers (Table 1) were used for qRT-PCR using the Platinum SYBR Green qPCR SuperMix UDG Kit (Invitrogen) and the ABI 7500 FAST system (Life Technologies).

\section{Chromatin immunoprecipitation (ChIP) assay}

The ChIP assay was performed as described previously ${ }^{3}$. In brief, $253 \mathrm{~J}$ cells were treated with $1 \%$ formaldehyde for 10 min to cross-link proteins with DNA. The cross-linked chromatin was then prepared and sonicated to an average size of 400-600 bp. The samples were diluted 10-fold and then precleared with protein A-agarose/salmon sperm DNA for $30 \mathrm{~min}$ at $4{ }^{\circ} \mathrm{C}$. The DNA fragments were immunoprecipitated overnight at $4{ }^{\circ} \mathrm{C}$ with anti-Runx1t1, anti-TCF4, or anti-IgG (as a negative control) antibody. After cross-linking reversal, TCF4 and Runx1t1 occupancy on the miR-625 promoter was examined. The results were determined by qRT-PCR. The ChIP primer sequences are summarized in Table 1.

\section{Target prediction}

TargetScan (http://www.targetscan.org/vert_72/), the miRanda database (http://www.microrna.org/microrna/ home.do) and RNA22 (http://cm.jefferson.edu/rna22/ Interactive//) ${ }^{23,24}$ were used to identify potential microRNAs targeting the $3^{\prime}$ UTR of RBM24.

\section{Statistical analysis}

Data are presented as the means \pm SEM. The Student's $t$-test was used to analyze the differences between two groups, and for multiple comparisons or repeated measurements, ANOVA or repeated ANOVA followed by Tukey's post hoc test was used. $P<0.05$ was considered statistically significant. Statistical analysis was performed using GraphPad Prism 7 software (GraphPad Software).

\section{Results}

\section{RBM24 is upregulated in BC tissues and contributes to} poor prognosis

Our previous study revealed that RBM5 is downregulated in $\mathrm{BC}$ tissue $^{3}$. In contrast, the mRNA and protein levels of RBM24 were frequently increased in BC tissue samples $(n=32)$ compared to normal bladder tissues $(n=32)$, as revealed by RT-qPCR and western blot analyses (Fig. 1a, b). Similar results were obtained by immunofluorescence staining with an RBM24-specific antibody in a cohort of $161 \mathrm{BC}$ specimens (Fig. 1c, d), and correlation analysis showed that the level of RBM24 was significantly associated with tumor size and stage but not with other clinicopathologic factors, such as age, sex, or tumor grade (Table 2). Additionally, the TCGA database also revealed that higher RBM24 mRNA levels in BC patients were associated with poor overall survival $(P=$ 0.00215 , Fig. 1e). Together, these clinical data suggest that upregulation of RBM24 may be a critical event driving BC progression.

\section{RBM24 promotes the proliferation of $B C$ cells in vitro}

To investigate the specific functions of RBM24 in BC, we first compared protein levels between a normal bladder cell line (SV-HUC-1) and a series of BC cell lines (UM-UC-3, 253 J, T24, and J82). RBM24 was significantly elevated in two tumor cell lines (UM-UC-3 and $253 \mathrm{~J}$ ) but downregulated in one (J82) compared to normal bladder cells (Fig. 2a). However, the mRNA expression of RBM24 was upregulated only in the $253 \mathrm{~J}$ cell line and decreased in the J82 cell line (Fig. 2b). Therefore, these two cell lines were selected for subsequent loss- and gain-of-function experiments. In light of the correlation between RBM24 expression and tumor stage, we speculated that RBM24 may be involved in cell proliferation. RBM24 knockdown in $253 \mathrm{~J}$ cells using a specific shRNA decreased RBM24 expression compared with the shRNA control vector, while RBM24 overexpression in J82 cells via transfection with a pWPI-RBM24 vector increased RBM24 expression compared with the empty overexpression vector. In addition, RBM24 knockdown in 253 J cells suppressed the expression of the cell proliferation marker CDK4, while RBM24 overexpression in J82 cells increased CDK4 expression (Fig. 2c). Furthermore, MTT and colony formation assays revealed that overexpression of RBM24 promoted J82 cell proliferation, while RBM24 knockdown suppressed the proliferation of $253 \mathrm{~J}$ cells (Fig. 2d, e). Together, these data suggest that RBM24 promotes BC cell proliferation.

\section{Runx1t1 mediates RBM24-induced cell proliferation}

Previous studies have reported that RBM24 functions as a regulator of multiple genes linked to cell proliferation, fate, differentiation, and apoptosis ${ }^{10}$. To investigate the molecular mechanisms underlying the regulation of $\mathrm{BC}$ cell proliferation by RBM24, we examined the expression levels of candidate effectors selected according to the previous studies ${ }^{10}$. Among the 6 candidate genes, Runx1t1 was significantly downregulated in RBM24-depleted $253 \mathrm{~J}$ cells and upregulated in RBM24-overexpressing J82 cells, as revealed by western blot analysis (Fig. 3a). Runx1t1 mRNA levels were significantly upregulated in BC tissue samples compared to normal bladder tissues as measured by RT-qPCR (Fig. 3b), and Runx1t1 protein levels were also significantly increased according to western blot and immunofluorescence staining (Fig. 3c, d). Moreover, human clinical data from the TCGA database of Oncolnc 


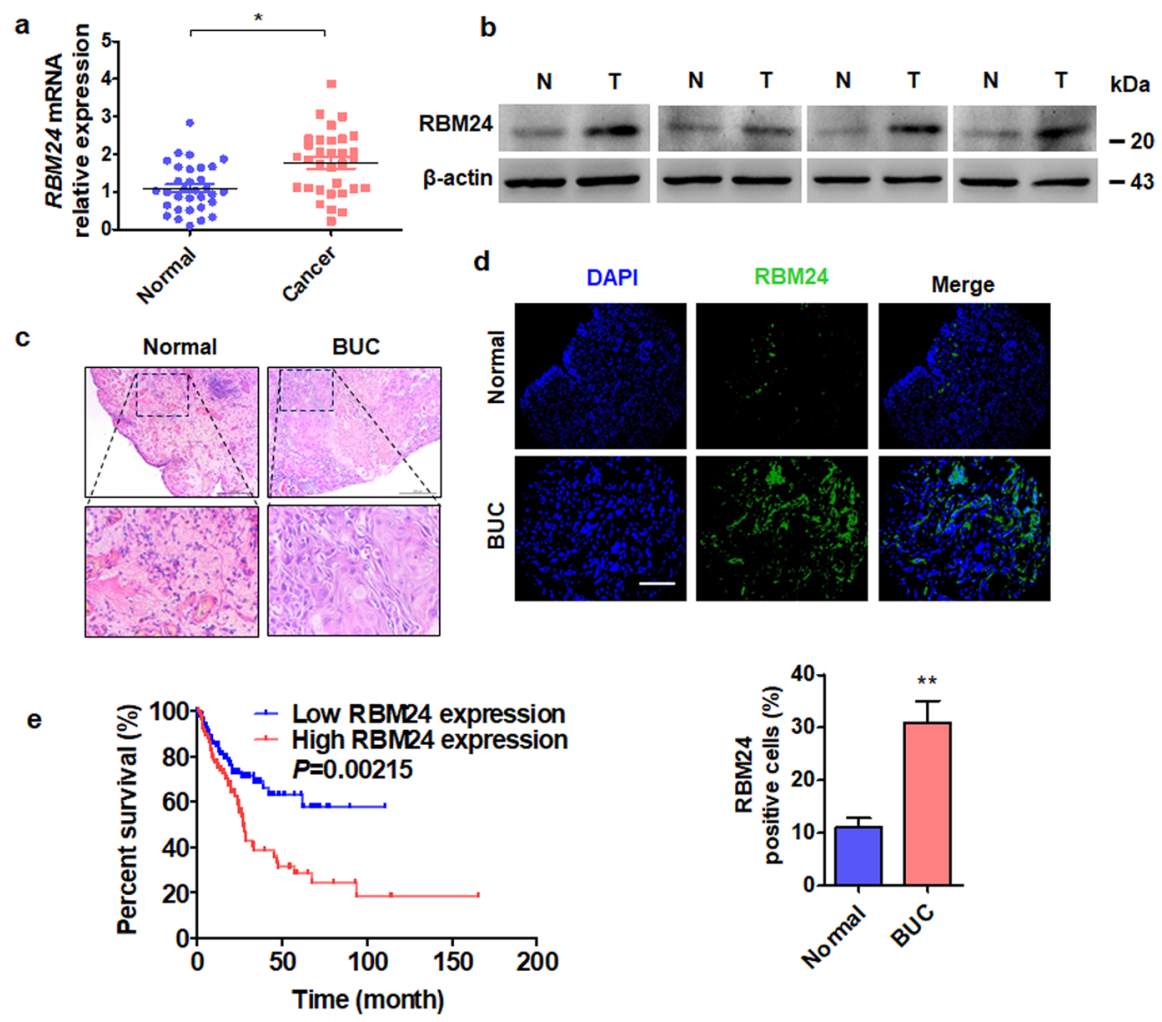

Fig. 1 RBM24 is upregulated in BC tissues and contributes to poor prognosis. a RT-qPCR was used to detect the expression level of RBM24 in BC tissues $(n=32)$ and normal bladder tissues $(n=32)$. ${ }^{P} P<0.05$ vs. normal bladder tissues. $\mathbf{b}$ Western blot analysis was used to examine the RBM24 protein level in four pairs of randomly selected tumor ( $T$ ) and normal bladder tissues ( $N$ ). c Hematoxylin and eosin staining of normal bladder and BC tissues. $\mathbf{d}$ Immunofluorescence staining of RBM24 in normal bladder and BC tissues. Scale bar $=50 \mu \mathrm{m}$. Bottom: statistical analysis of the percentage of RBM24-positive cells. e Kaplan-Meier analysis was used to analyze the overall survival of BC patients with low $(n=130)$ or high RBM24 ( $n=130)$ levels from the TCGA database (cutoff value $=25 \%$ ).

(http://www.oncolnc.org) revealed that higher Runx1t1 expression was associated with poor prognosis $(P=$ 0.0064, Fig. 3e). Additionally, correlation analysis showed that RBM24 mRNA expression was positively correlated with Runx1t1 mRNA expression in BC tissue (Fig. 3f).

To determine whether Runx1t1 contributes to RBM24regulated $B C$ cell proliferation, we performed a rescue experiment. As shown in Fig. 3g, $253 \mathrm{~J}$ cell growth was markedly reduced after combined knockdown of RBM24 and Runx1t1 compared to that after knockdown of either gene alone. Conversely, the enhanced proliferation observed in 582 cells overexpressing RBM24 was abolished by cotransfection with shRunx1t1, as evidenced by colony formation assay (Fig. 3h). Together, these data suggest that Runx1t1 participates in the RBM24-mediated regulation of $\mathrm{BC}$ cell proliferation.

\section{RBM24 promotes Runx1t1 expression by stabilizing its mRNA}

Because RBM24 and Runx1t1 were positively correlated in $\mathrm{BC}$, we then investigated whether RBM24 regulates Runx1t1 expression in BC cells and the underlying mechanisms. RT-qPCR results showed that overexpression of RBM24 upregulated the mRNA expression level of Runx1t1, while knockdown of RBM24 downregulated the mRNA expression level of Runx1t1 in BC cells (Fig. 4a). As previous studies have reported that RBM24 enhances RNA stability ${ }^{10,25}$, we investigated whether elevated RBM24 prolongs Runx1t1 mRNA expression following blockade of new transcription using actinomycin D (ActD). Indeed, RBM24 knockdown in $253 \mathrm{~J}$ cells reduced the duration of Runx1t1 mRNA expression, whereas RBM24 overexpression in J82 cells prolonged Runx1t1 mRNA expression, consistent with RBM24-mediated enhancement of Runx1t1 mRNA stability (Fig. 4b). To confirm this notion, we examined whether RBM24 binds to Runx1t1 mRNA using in vitro RNA pull-down and RNA-binding protein immunoprecipitation (RIP) assays. Coimmunoprecipitation confirmed that the RBM24 antibody effectively pulled down endogenous RBM24 protein (Fig. 4c), and PCR analysis of RIP products revealed the presence of the STAT3 and Runx1t1 mRNA $3^{\prime}$-untranslated regions ( $3^{\prime}$ UTRs) but not the $3^{\prime}$ UTR of GAPDH (Fig. 4d). Consistent with this 
Table 2 Correlations between RBM24 mRNA expression and clinicopathological characteristics.

\begin{tabular}{|c|c|c|c|c|}
\hline \multirow[t]{2}{*}{ Characteristic } & \multirow{2}{*}{$\begin{array}{l}\text { Number of } \\
\text { patients (\%) }\end{array}$} & \multicolumn{2}{|c|}{ RBM24 expression } & \multirow[t]{2}{*}{$P$-value ${ }^{\mathrm{a}}$} \\
\hline & & Low (\%) & High (\%) & \\
\hline $\begin{array}{l}\text { Total no. of } \\
\text { patients }\end{array}$ & 161 & 89 & 72 & \\
\hline \multicolumn{5}{|l|}{ Age (years) } \\
\hline$\leq 63^{b}$ & 70 & $37(52.86)$ & $33(47.14)$ & 0.772 \\
\hline$>63$ & 91 & $46(50.55)$ & $45(49.45)$ & \\
\hline \multicolumn{5}{|l|}{ Gender } \\
\hline Male & 102 & $56(54.90)$ & $46(45.10)$ & 0.264 \\
\hline Female & 59 & $27(45.76)$ & $32(54.24)$ & \\
\hline \multicolumn{5}{|c|}{ Tumor size $(\mathrm{cm})$} \\
\hline$\leq 3.0^{c}$ & 106 & $58(54.72)$ & $48(45.28)$ & 0.001 \\
\hline$>3.0$ & 55 & $15(27.27)$ & $40(72.73)$ & \\
\hline \multicolumn{5}{|l|}{ Tumor grade } \\
\hline Low & 97 & $45(46.39)$ & $52(53.61)$ & 0.221 \\
\hline High & 64 & $36(56.25)$ & $28(43.75)$ & \\
\hline \multicolumn{5}{|l|}{$T$ classification } \\
\hline $\mathrm{Ta}, \mathrm{T} 1$ & 105 & $46(43.81)$ & $59(56.19)$ & 0.041 \\
\hline T2-T4 & 56 & $34(60.71)$ & $22(39.29)$ & \\
\hline \multicolumn{5}{|l|}{$p N$ status } \\
\hline $\mathrm{pN}-$ & 114 & $55(48.25)$ & $59(51.75)$ & 0.120 \\
\hline $\mathrm{pN}+$ & 47 & $29(61.70)$ & $18(38.30)$ & \\
\hline \multicolumn{5}{|c|}{ Tumor multiplicity } \\
\hline Unifocal & 69 & $28(40.58)$ & $41(59.42)$ & 0.818 \\
\hline Multifocal & 92 & $39(42.39)$ & $53(57.61)$ & \\
\hline
\end{tabular}

Significant associations are shown in bold in the $p$-value column $(p$-value $<0.05)$. ${ }^{\text {a }}$ Chi-square test.

${ }^{\mathrm{b}}$ Median age.

cMedian size.

finding, a biotinylated Runx1t1 mRNA-3'UTR and positive control STAT3-mRNA probe pulled down RBM24 protein, while a GAPDH mRNA-3'UTR probe did not (Fig. 4e). These results suggest that RBM24 can bind to the Runx1t1 mRNA 3'UTR, thereby enhancing mRNA stability.

We then used western blotting to determine whether Runx1t1 regulates the expression levels of genes associated with cell proliferation. Depletion of Runx1t1 in $253 \mathrm{~J}$ cells reduced the expression of the proliferation marker gene CDK4, while overexpression of Runx1t1 in J82 cells increased CDK4 protein levels (Fig. 4f). While RBM24 protein expression was positively regulated by Runx1t1 protein expression, neither depletion of Runx1t1 in $253 \mathrm{~J}$ cells nor overexpression of Runx1t1 in J82 cells altered RBM24 mRNA levels (Fig. 4g).

\section{Runx1t1 interacts with the transcription factor TCF4 to mediate RBM24 upregulation and cell proliferation}

Runx1t1 functions as a transcription cofactor by interacting with various partner proteins ${ }^{26,27}$. To identify the partner(s) involved in RBM24 regulation, we performed coimmunoprecipitation coupled with mass spectrometry (CoIP-MS). Eight proteins were upregulated in RBM24overexpressing $\mathrm{BC}$ cells, including the transcription factor TCF4 (Fig. 5a), and reciprocal immunoprecipitation showed a strong interaction between Runx1t1 and TCF4 in $\mathrm{BC}$ cells (Fig. 5b). Additionally, an in situ proximity ligation assay (PLA) confirmed direct binding between Runx1t1 and TCF4 (Fig. 5c). In addition, TCF4 mRNA levels were significantly upregulated in BC tissues compared to normal bladder tissues (Fig. 5d) and positively correlated with Runx1t1 mRNA levels (Fig. 5e). Analysis of survival data from the TCGA database showed that higher expression of TCF4 was associated with poor prognosis (Fig. 5f).

To investigate whether TCF4 participates in the RBM24/Runx1t1 axis to regulate BC cell proliferation, we performed rescue experiments. Cotransfection of 253 J cells with shTCF 4 and shRunx1t1 inhibited the expression of RBM24 and CDK4 to a greater extent than knockdown of Runx1t1 alone (Fig. 5g). Transfection of J82 cells with shTCF4 also sharply reduced RBM24 and CDK4 expression levels, and this reduction was reversed by cotransfection with the Runx1t1 overexpression vector (Fig. 5h). Knockdown of TCF4 and Runx1t1 also decreased the proliferation rate of $253 \mathrm{~J}$ cells to a greater extent than knockdown of either protein alone. Conversely, overexpression of Runx1t1 promoted J82 cell growth, an effect that was abolished by shTCF4 cotransfection (Fig. 5i). Since Runx1t1 alone did not regulate RBM24 transcription (Fig. 4g), we examined whether TCF4 regulates RBM24 transcription. However, neither Runx1t1 nor TCF4 regulated RBM24 mRNA expression or modulated the stability of the RBM24 protein (Fig. 5j, k, Supplementary Fig. 1). Together, these data suggest that Runx1t1 interacts with TCF4 to promote RBM24 protein expression rather than gene transcription.

\section{miR-625-5p mediates Runx1t1/TCF4-regulated proliferation by direct targeting in $\mathrm{BC}$ cells}

Our finding that Runx1t1/TCF4 regulates RBM24 protein expression without influencing RBM24 mRNA levels suggests that Runx1t1/TCF4 regulates RBM24 at the posttranscriptional level. MicroRNAs (miRNAs) are critical regulators of gene expression at the posttranscriptional level, so we conducted bioinformatics analyses using TargetScan, miRanda, and RNA22 to predict 


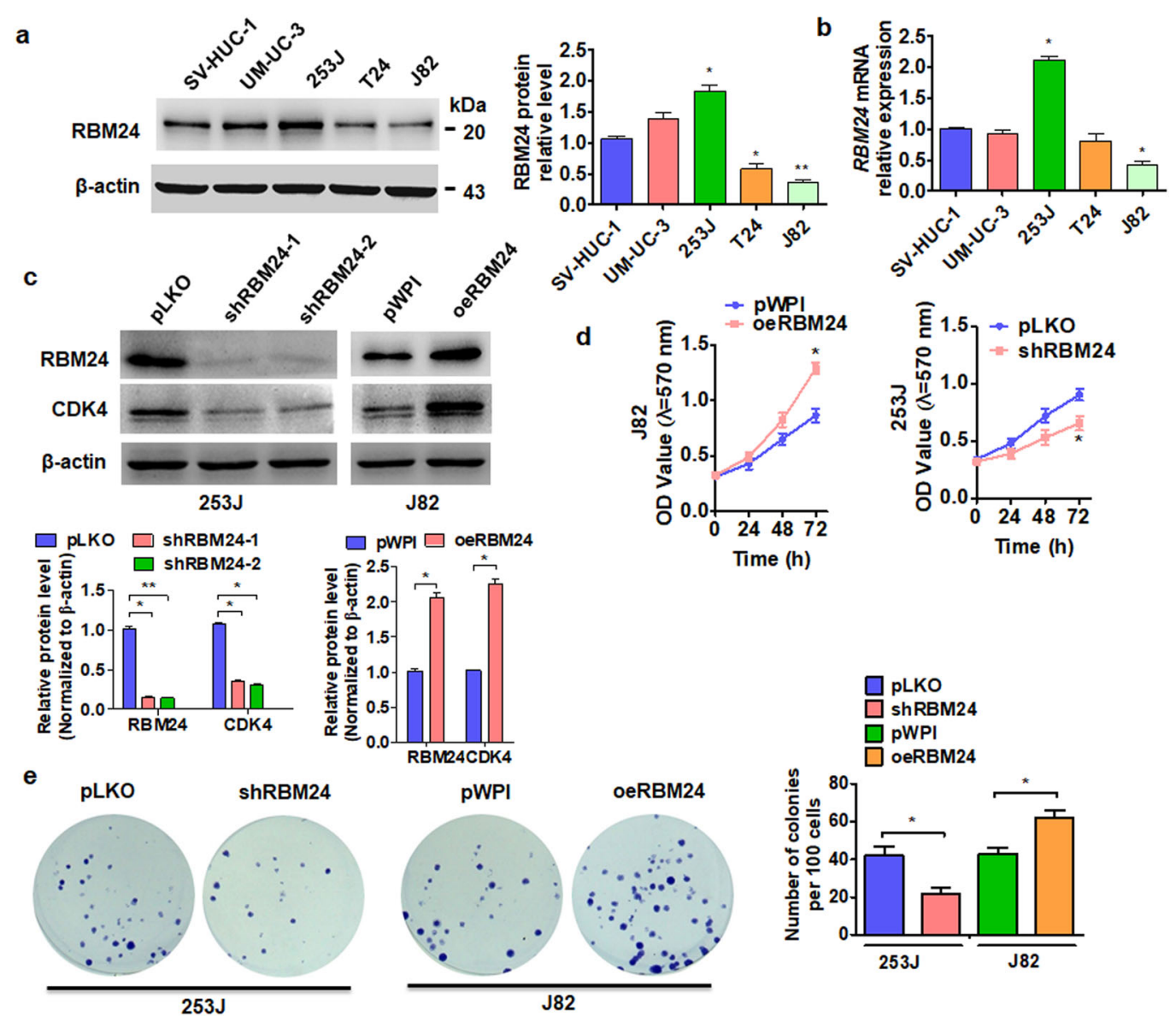

Fig. 2 RBM24 promotes the proliferation of BC cells in vitro. a Western blot analysis was used to examine the protein level of RBM24 in human uroepithelial cells (SV-HUC-1) and BC cell lines (UM-UC-3, 253 J, T24, and J82). Densitometric analysis (A) was performed in three independent experiments. ${ }^{*} P<0.05$, ${ }^{* *} P<0.01$ vs. the SV-HUC-1 group. $\mathbf{b}$ RT-qPCR was used to detect the mRNA expression of RBM 24 in the cell lines. ${ }^{*} P<0.05$ vs. the SV-HUC-1 group. $\mathbf{c}$ The RBM24 and CDK4 protein levels were measured by western blot analysis in shRBM24-transfected $253 \mathrm{~J}$ cells or RBM24 overexpression vector (oeRBM24)-transfected $J 82$ cells. ${ }^{*} P<0.05,{ }^{* *} P<0.01$ vs. the corresponding control. $\mathbf{d}$ Cells were prepared as in (c), and cell viability was measured by MTT assay. ${ }^{*} P<0.05$ vs the corresponding control. e Cells were prepared as in (c), and cell viability was measured by colony formation assays. The right panel shows the numbers of colonies formed. ${ }^{*} P<0.05$ vs the corresponding control.

miRNA sequences targeting the RBM24 3'UTR and then used a T7 RNA transcriptase to generate an RBM24 $3^{\prime}$ UTR containing biotin-labeled uracil (Fig. 6a). After transfection, complementary miRNAs were extracted by pull-down assay, and the expression levels of 9 candidates were compared by RT-PCR. The results showed that miR149-3p, miR-216a-5p, miR-625-5p, miR-449a, and miR578 were enriched by the RBM24 3'UTR pull-down assay (Fig. 6b). Next, we assessed miRNA expression levels after up- and downregulation of Runx1t1 and TCF4 expression to identify the miRNAs involved in Runx1t1/TCF4mediated regulation of RBM24 expression. As shown in Fig. 6c, only miR-625-5p was regulated by both Runx1t1 and TCF4. Furthermore, a luciferase reporter assay showed that miR-625-5p directly targeted the RBM24 $3^{\prime}$ UTR (Fig. 6d, e), and western blot analysis confirmed that
RBM24 protein expression was reduced by miR-625-5p mimic transfection into BC cells compared to transfection with its antagomir (Fig. 6f). Notably, the expression of miR-625-5p was downregulated in BC tissue compared to normal bladder tissue (Fig. 6g). Moreover, analysis of survival data from the TCGA database revealed that lower expression of miR-625-5p was associated with poor prognosis (Fig. 6h). Additionally, miR-625-5p expression was negatively correlated with RBM24 mRNA expression in $\mathrm{BC}$ tissue (Fig. 6i).

To investigate whether the Runx1t1/TCF4 complex regulates RBM24 expression by directly suppressing miR-625-5p transcription, we first predicted the potential TCF element within the 2-kb $5^{\prime}$-promoter region of miR-625-5p using the Ensembl and PROMO 3.0 websites, which identified three potential TCF 


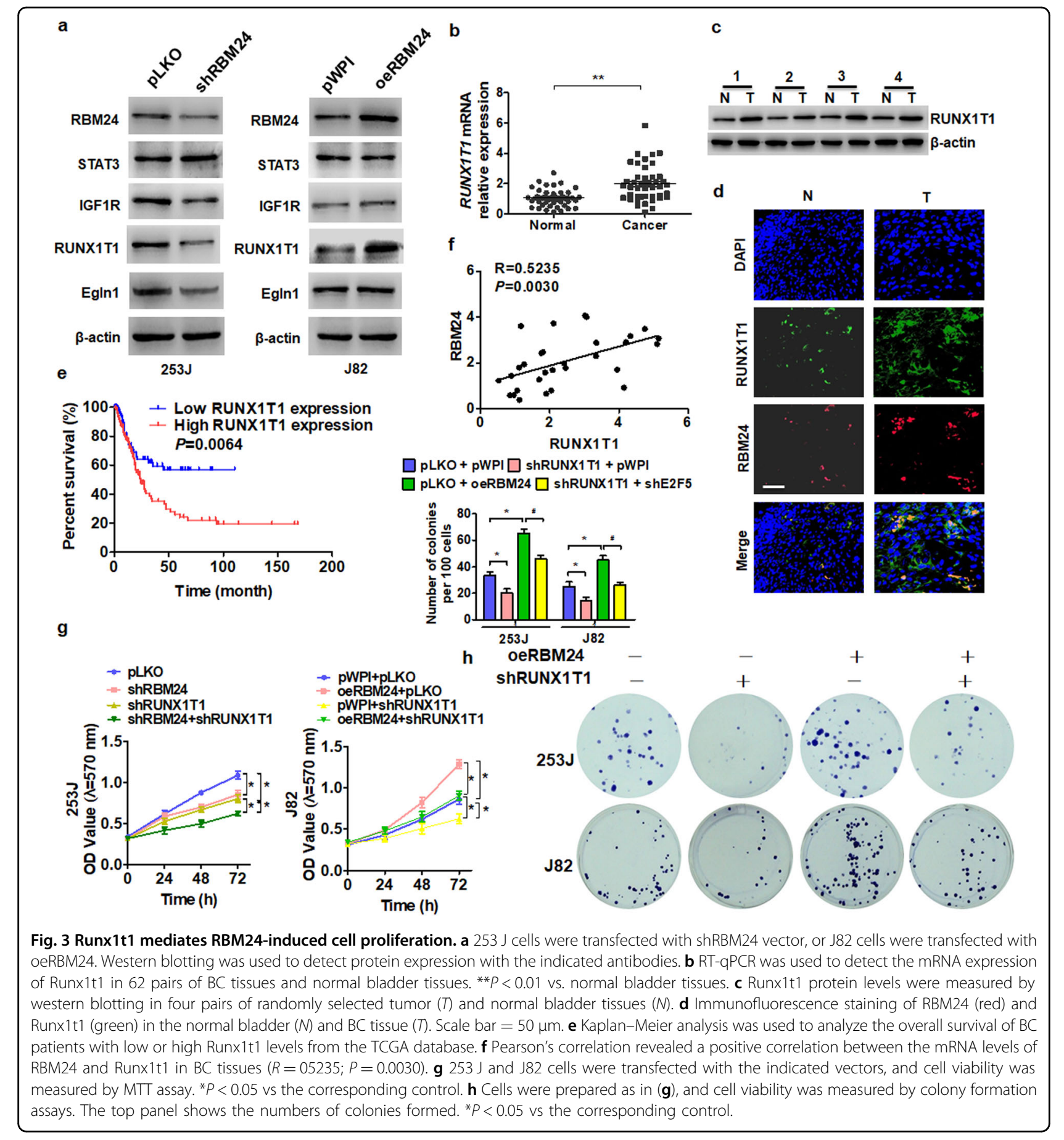

elements (Fig. 6j). ChIP analysis confirmed that Runx1t1/TCF4 bound predominantly to the region located -522 to $-532 \mathrm{bp}$ upstream of the transcription start site within the miR-625-5p promoter (Fig. 6k), and a luciferase assay yielded similar results (Fig. 6l). Together, these findings indicate that Runx1t1/TCF4 directly inhibits the transcription of miR-625-5p, thereby disinhibiting RBM24 expression.
Disruption of the RBM24/Runx1t1/TCF4/miR-625-5p axis inhibits $B C$ xenograft growth in vivo

Finally, we examined whether RBM24 and Runx1t1 regulate $\mathrm{BC}$ cell growth in vivo using a xenograft model. Injection of $253 \mathrm{~J}$ cells with stable knockdown of RBM24 and Runx1t1 yielded smaller tumors in nude mice than the injection of sham-transfected $253 \mathrm{~J}$ cells. Furthermore, the tumor volume was much smaller in mice 


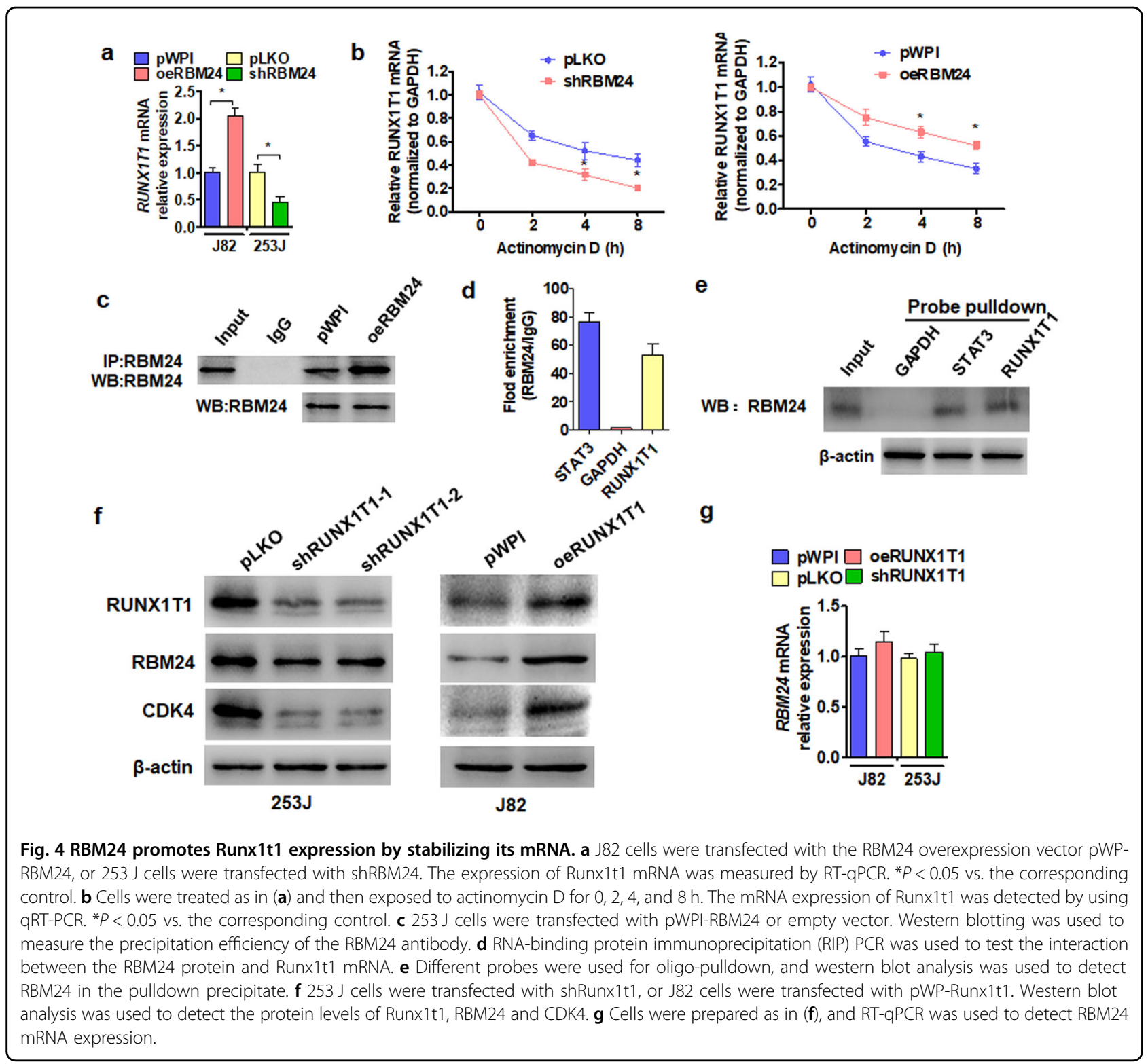

implanted with RBM24/Runx11 double knockdown cells than in mice implanted with single knockdown cells (Fig. $7 \mathrm{a}, \mathrm{b})$. These findings were mirrored by the mean tumor wet weights following excision (Fig. 7c). Western blot analysis of extracted tumor tissue also demonstrated that tumors derived from cells with silencing of either Runx1t1 or RBM24 exhibited significantly downregulated Runx1t1, RBM24, and CDK4 levels compared to tumors derived from control cells, and this downregulation was further enhanced by the simultaneous knockdown of both Runx1t1 and RBM24 (Fig. 7d). These findings suggest that the RBM24/Runx1t1/TCF4/ miR-625-5p axis inhibits the proliferation of $\mathrm{BC}$ cells in vivo.

\section{Discussion}

In this study, we identified an RBM24/Runx1t1/TCF4/ miR-625-5p feedback loop that drives BC progression. First, RBM24 expression was significantly higher in $B C$ tissues than in corresponding normal tissues, and elevated RBM24 expression was correlated with poor prognosis. Second, RBM24 overexpression promoted BC cell proliferation in vivo and in vitro and enhanced Runx1t1 protein expression by increasing Runx1t1 mRNA stability. Third, Runx1t1 interacted with the transcription factor TCF4, and this Runx1t1-TCF4 complex positively regulated RBM24 protein expression by suppressing the expression of the RBM24 negative regulator miR-625-5p, resulting in the formation of a positive feedback loop 


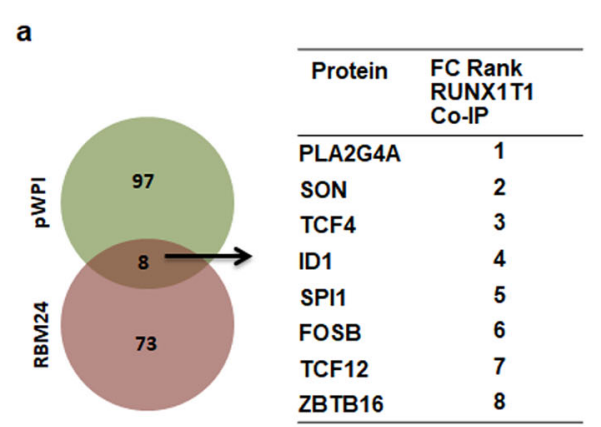

d

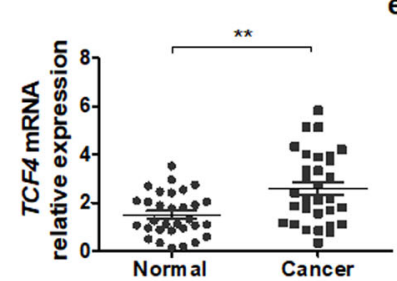

b

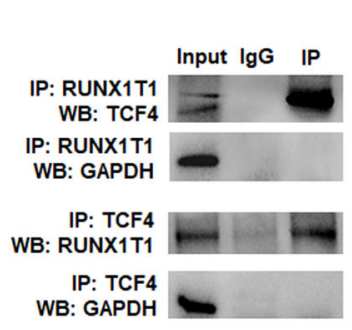

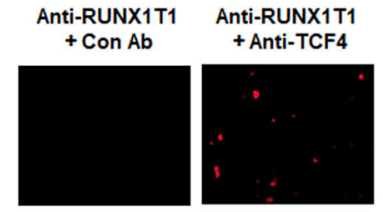
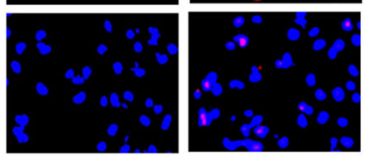

f

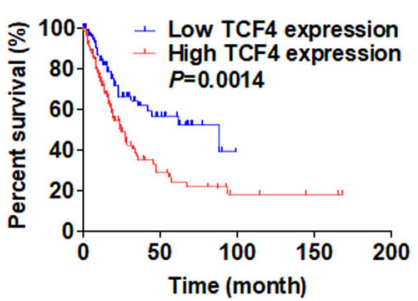

h

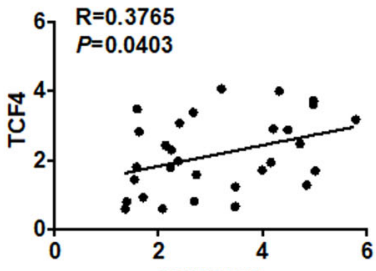

RUNX1T1 i

g

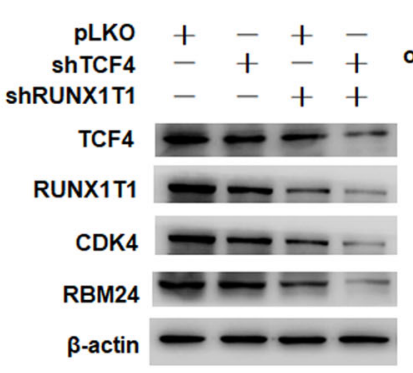

253J
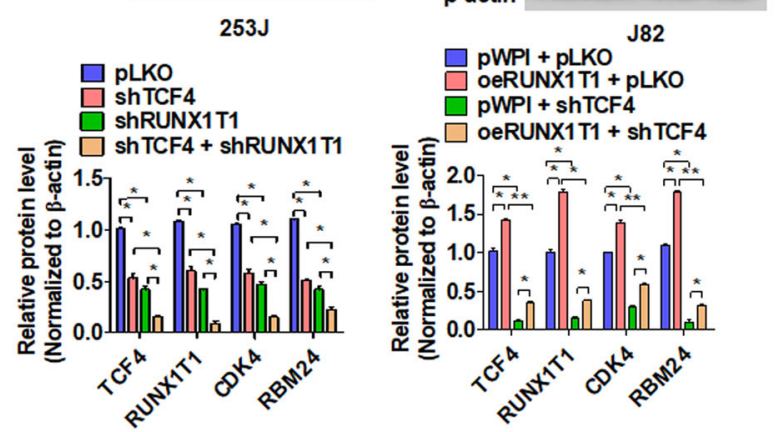

i $\quad$ pLKO

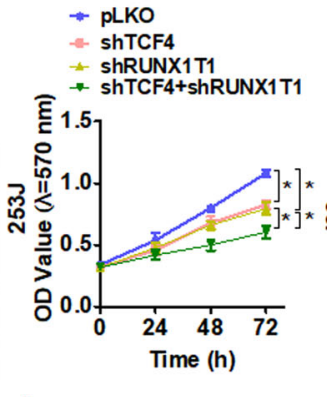

j
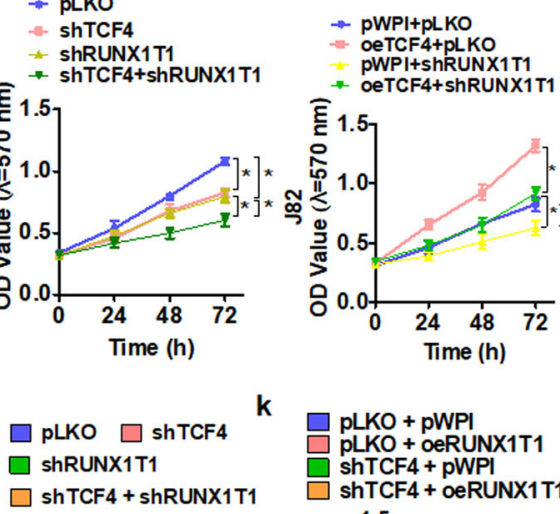

- PWPI+ShRUNX1T1

$\widehat{E}$

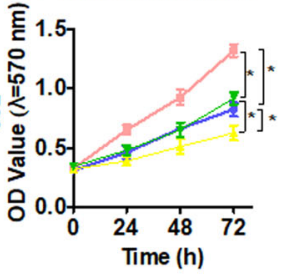

k

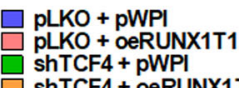

$\square$ shTCF4 + shRUNX1T1 ShTCF4 + pWPI

$\square$ shTCF4 + oeRUNX1T1
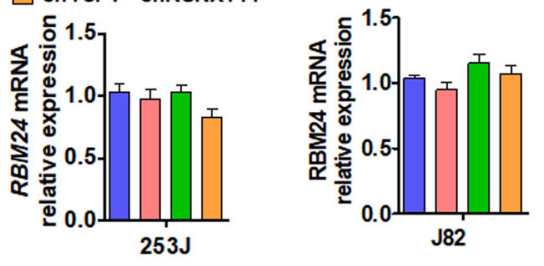

Fig. 5 Runx1t1 interacts with the transcription factor TCF4 to mediate RBM24 upregulation and cell proliferation. a Coimmunoprecipitation coupled with mass spectrometry (ColP-MS) was performed with an anti-Runx1t1 antibody in J82 cells to determine whether the proteins interacting with Runx 1 t 1 change after RBM24 overexpression. The right table shows 8 proteins that exhibited increased interaction with Runx $1 \mathrm{t} 1$. b ColP analysis was used to detect the interaction between TCF4 and Runx1t1, and GAPDH was used as a negative control. $\mathbf{c}$ In situ proximity ligation analysis (PLA) detected the interaction between TCF4 and Runx1t1. Red color indicates PLA-positive cells. $\mathbf{d}$ RT-qPCR was used to examine the expression of TCF4 in BC tissues and normal bladder tissues. ${ }^{*} P<0.01$ vs. normal bladder tissues. e Pearson's correlation analysis of the correlation between TCF4 and Runx1t1 mRNAs in $B C$ tissues $(R=0.3765 ; P=0.0403)$. $\mathbf{f}$ Kaplan-Meier analysis was used to analyze the overall survival of $\mathrm{BC}$ patients with low or high TCF4 levels from TCGA database $(P=0.0014)$. $\mathbf{g}$, $\mathbf{h}$ Western blot analysis was used to examine TCF4, Runx1t1, CDK4 and RBM24 expression in $253 \mathrm{~J}$ cells transfected with shTCF4 and shRunx1t1 either alone or together (g) or J82 cells transfected with oeRunx1t1 and shTCF4 either alone or together (h). The attached panel shows the densitometric analysis from three independent experiments. ${ }^{*} P<0.05,{ }^{*} P<0.01$ vs. the corresponding control. i Cells were prepared as in $(\mathbf{g}, \mathbf{h})$, and cell viability was measured by MTT assay. ${ }^{*} P<0.05$ vs. the corresponding control. $\mathbf{j}$, $\mathbf{k}$ Cells were prepared as in $(\mathbf{g}, \mathbf{h})$, and RT-qPCR was used to detect the mRNA expression of RBM24. 
a
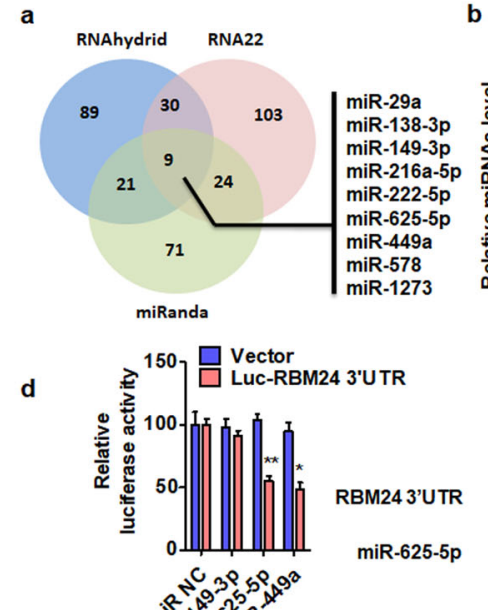

b

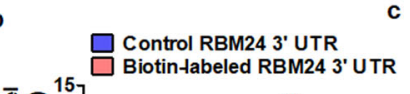

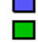
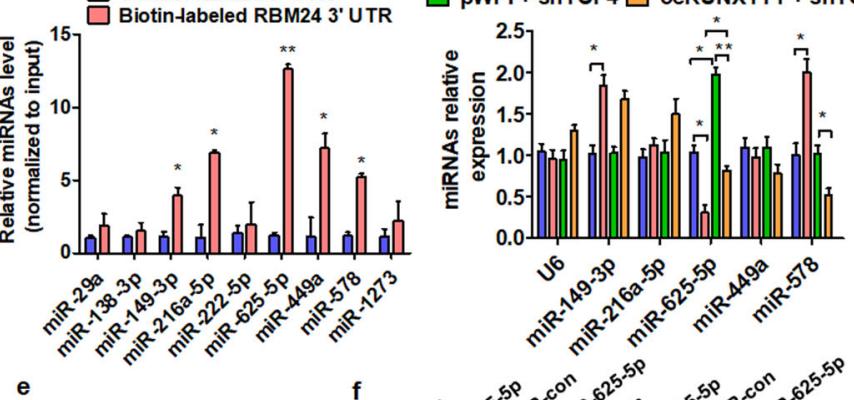

g

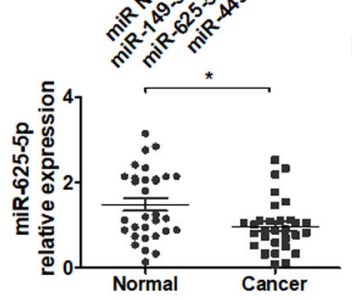

h

GAATTGTTTTCTCTTTCOCTOC

$|:|:|\quad||||||||:|$

GAAAGGGGA

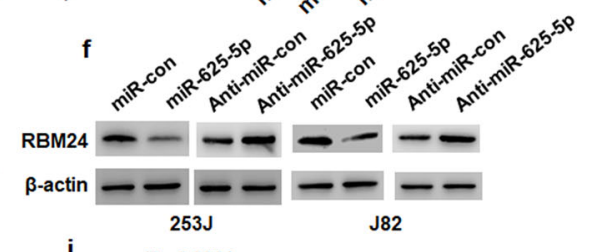

i $R=-0.3901$

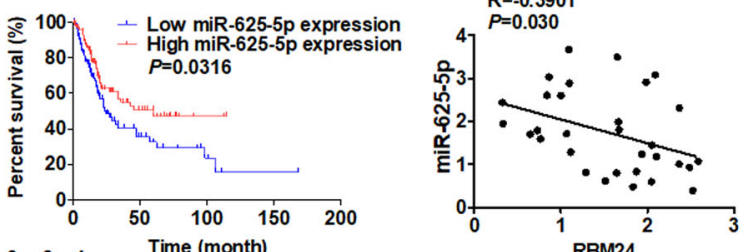

j

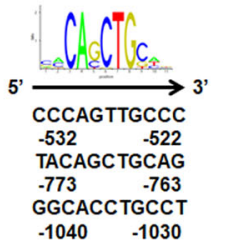

k

$3,2<1$
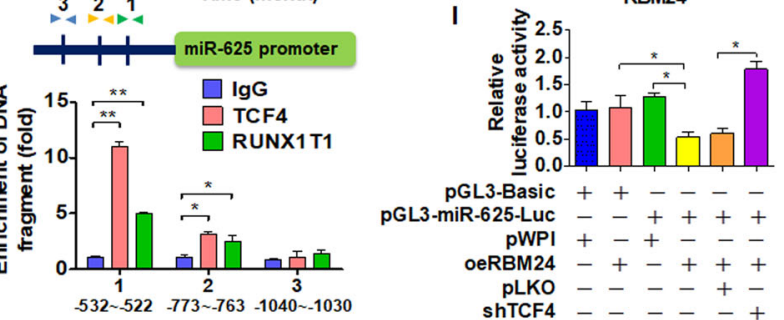

pGL3-Basic $++\ldots$

pGL3-miR-625-Luc --+++

pWPI +-+---

oeRBM24 - + + + +

pLKO ----+

Fig. 6 miR-625-5p mediates Runx1t1/TCF4-regulated proliferation by direct targeting in BC cells. a Venn diagram displaying potential microRNAs associated with the RBM24 $3^{\prime} U T R$ sequence from three online target-prediction programs. b Biotin-labeled RBM24 $3^{\prime} U T R R$ RNA was transfected into J82 cells, followed by a biotin pull-down assay using Streptavidin-coupled Dynabeads. The miRNAs were extracted from the sedimented beads, and the relative levels of 9 candidate miRNAs were detected by RT-qPCR. ${ }^{*} P<0.05,{ }^{* *} P<0.01$ vs. the control probe. c J82 cells were transfected with oeRunx1t1 or shTCF4 or cotransfected with both vectors together. RT-qPCR was used to detect the expression of 5 miRNAs. ${ }^{*} P<0.05$, ${ }^{*} P<0.01$ vs. the corresponding controls. $\mathbf{d} \mathrm{J} 82$ cells were cotransfected with the RBM24 $3^{\prime} U T R$ and the indicated miRNA mimics. Luciferase reporter assays showed that miR-625-5p and miR-449a reduced RBM24 $3^{\prime}$ UTR luciferase activity. ${ }^{*} P<0.05,{ }^{*} P<0.01$ vs. the control vector. e The predicted miR-625-5p binding site in the RBM24 $3^{\prime} U$ TR. $\mathbf{f} 253 \mathrm{~J}$ and $\mathrm{J} 82$ cells were transfected with the indicated miRNAs, and then western blotting was used to detect RBM24 protein expression. $\mathbf{g}$ RT-qPCR was used to detect the expression of miR-625-5p in BC tissues or normal bladder tissues. ${ }^{*} P<0.05$ vs. normal bladder tissues. $\mathbf{h}$ Kaplan-Meier analysis of data from the TCGA database was used to analyze the correlation between the overall survival of BC patients with miR-625-5p levels $(P=0.0316)$. i Pearson's correlation analysis of the correlation between RBM24 and miR-625-5p expression in $\mathrm{BC}$ tissues $(R=-0.3901 ; P=0.0030)$. $\mathbf{j}$ Potential binding site of TCF4 in the miR-625 promoter. $\mathbf{k}$ ChIP-qPCR was used to detect TCF4 and Runx1t1 complex binding to the miR-625 promoter region in 293 A cells. ${ }^{*} P<0.05,{ }^{* *} P<0.01$ vs. IgG. I The miR-625 promoter-luciferase reporter was cotransfected with the RBM24 overexpression vector or with the shTCF4 vector into 293 A cells. Luciferase reporter assays were performed to determine luciferase activity. ${ }^{*} P<0.05$ vs. the corresponding control.

driving elevated RBM24 expression and BC cell proliferation. These findings suggest that the RBM24/ Runx1t1/TCF4/miR-625-5p axis is a critical promoter of $\mathrm{BC}$ initiation and progression (Fig. 7e).

RBPs influence the structure and interactions of target RNAs, thereby influencing RNA biogenesis, stability, function, transport, and subcellular localization ${ }^{28}$. Many RBPs are expressed in a tissue-specific manner to drive developmental processes ${ }^{29}$. For instance, RBM24 is highly expressed in heart and muscle tissues ${ }^{7}$ and regulates cardiac embryonic stem cell differentiation via a splicingmediated mechanism ${ }^{30}$. In addition, RBM24 was reported to suppress nasopharyngeal cancer progression ${ }^{9}$. In the current study, however, RBM24 accelerated BC cell proliferation both in vivo and in vitro. This discrepancy may be explained by differences in upstream and downstream 


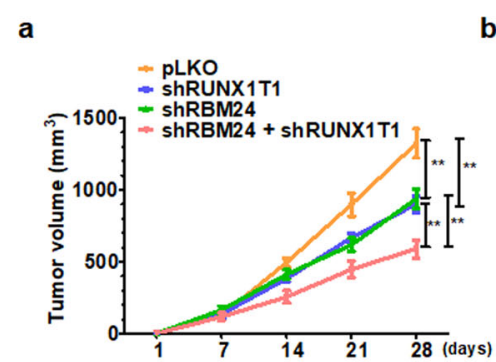

d
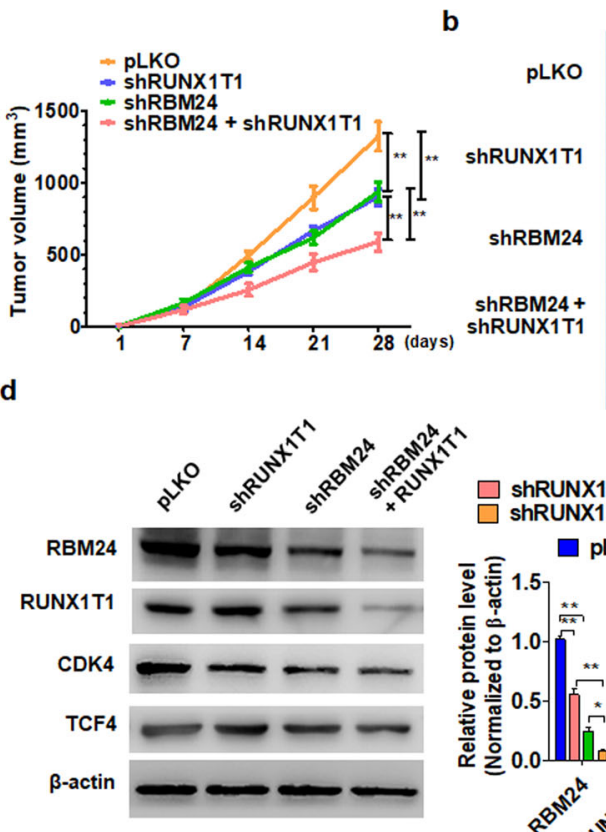

\section{,}

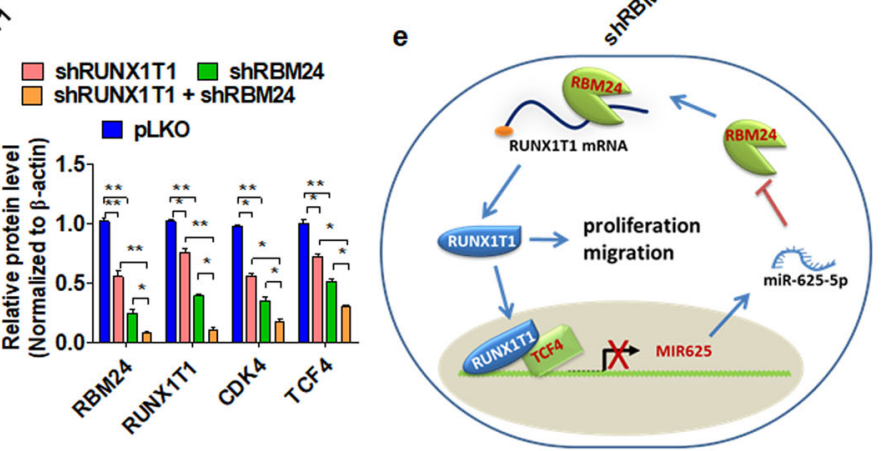

Fig. 7 Disruption of the RBM24/Runx1t1/TCF4/miR-625-5p axis inhibits BC xenograft growth in vivo. a $253 \mathrm{~J}$ cells with stable depletion of RBM24, Runx1t1, or both together were injected subcutaneously into nude mice to establish BC xenograft tumors. Tumor volumes were monitored by direct measurement. ${ }^{*} P<0.05$, ${ }^{*} P<0.01$ vs. the corresponding control. b Representative tumor sizes in each group of mice. $\mathbf{c}$ Xenograft tumor wet weight in each group of mice. ${ }^{*} P<0.05,{ }^{*} P<0.01$ vs. the corresponding control. $\mathbf{d}$ Western blot analysis was used to measure the protein levels of RBM24, Runx1t1, CDK4, and TCF4 in xenograft tumors.

regulatory factors ${ }^{31}$. Consistent with this accelerated proliferation, high levels of RBM24 promoted the expression of Runx1t1 and correlated with poor prognosis in $\mathrm{BC}$ patients.

Transcription factor 4 (TCF4) is a member of the helix-loop-helix (bHLH) transcription factor family that recognizes and binds the Ephrussi box (E-Box) DNA element $\left(5^{\prime} \text {-ACANNTGT-3' }\right)^{32}$. TCF4 regulates chromatin remodeling and transcription by recruiting histone acetyltransferases (HATs), such as $\mathrm{p} 300^{21}$. Numerous studies have also implicated TCF4 in cancer progression. Jagrut et al. reported that TCF4 signaling was upregulated in colon cancer stem cells and promoted growth and self-renewal ${ }^{33}$. The expression of TCF4 is associated with breast cancer chemoresistance ${ }^{34}$. Additionally, high expression of TCF4 was an independent adverse prognostic factor in acute myeloid leukemia ${ }^{35}$. However, the expression and function of TCF4 in BC are largely unknown. In the present study, we found that TCF4 was upregulated in BC tissues and that high TCF4 expression was predictive of a poor prognosis. We demonstrated that interaction with Runx1t1 and subsequent downregulation of miR-625-5p, resulting in RBM24 upregulation, is one mechanism underlying the oncogenic effect of TCF4.

\section{Conclusion}

The present study demonstrates that upregulation of RBM24 enhances BC cell proliferation and leads to poor BC prognosis by initiating a Runx1t1/TCF4/miR-625-5p feedback loop. These findings highlight the RBM24/Runx1t1/ TCF4/miR-625-5p axis as a potential therapeutic target for $\mathrm{BC}$ treatment.

\section{Author contributions}

Conception and design: Y.Y., K.L. and P.G.; tissue collection: B.L., Y.N. and C.Z; development of methodology: Y.Y., B.L., J.C. and P.G.; acquisition of data: Y.Y., W.L. and K.L.; analysis of data: W.L., Y.Y. and J.C.; and writing the manuscript: Y. Y., K.L. and J.C.

\section{Conflict of interest}

The authors declare no competing interests.

Ethics approval and consent to participate

The present study was authorized by the Ethics Committee of Hebei Medical University. All patients and volunteers were anonymous and provided written informed consent.

\section{Publisher's note}

Springer Nature remains neutral with regard to jurisdictional claims in published maps and institutional affiliations.

Supplementary information The online version contains supplementary material available at https://doi.org/10.1038/s12276-021-00623-w. 
Received: 7 September 2020 Revised: 31 January 2021 Accepted: 23 February 2021.

Published online: 21 May 2021

\section{References}

1. Martinez Rodriguez, R. H., Buisan Rueda, O. \& Ibarz, L. Bladder cancer: Present and future. Med Clin.149, 449-455 (2017).

2. Bhanvadia, S. K. Bladder Cancer Survivorship. Curr. Urol. Rep. 19, 111 (2018).

3. Zhang, Y. P. et al. Down-regulated RBM5 inhibits bladder cancer cell apoptosis by initiating an miR-432-5p/beta-catenin feedback loop. FASEB J. 33, 10973-10985 (2019).

4. Dy, G. W., Gore, J. L., Forouzanfar, M. H., Naghavi, M. \& Fitzmaurice, C. Global burden of urologic cancers, 1990-2013. Eur. Urol. 71, 437-446 (2017).

5. Gaertner, A., Brodehl, A. \& Milting, H. Screening for mutations in human cardiomyopathy- is RBM24 a new but rare disease gene? Protein Cell 10, 393-394 (2019).

6. Yang, J. et al. RBM24 is a major regulator of muscle-specific alternative splicing. Dev. Cell 31, 87-99 (2014).

7. Poon, K. L. et al. RNA-binding protein RBM24 is required for sarcomere assembly and heart contractility. Cardiovasc. Res 94, 418-427 (2012).

8. $\mathrm{Xu}, \mathrm{E}$. et al. RNA-binding protein RBM24 regulates p63 expression via mRNA stability. Mol. Cancer Res. 12, 359-369 (2014)

9. Hua, W. F. et al. RBM24 suppresses cancer progression by upregulating miR-25 to target MALAT1 in nasopharyngeal carcinoma. Cell Death Dis. 7, e2352 (2016).

10. Lin, Y. et al. Global profiling of Rbm24 bound RNAs uncovers a multi-tasking RNA binding protein. Int. J. Biochem. Cell Biol. 94, 10-21 (2018).

11. Amann, J. M. et al. ETO, a target of $t(8 ; 21)$ in acute leukemia, makes distinct contacts with multiple histone deacetylases and binds mSin3A through its oligomerization domain. Mol. Cell Biol. 21, 6470-6483 (2001).

12. Miyoshi, $H$. et al. The $t(8 ; 21)$ translocation in acute myeloid leukemia results in production of an AML1-MTG8 fusion transcript. EMBO J. 12, 2715-2721 (1993).

13. J Nathan Davis, Laura McGhee, Shari Meyers. The ETO (MTG8) gene family. Gene. 2003 Jan 16;303:1-10. https:/doi.org/10.1016/s0378-1119(02)01172-1.

14. Cheng, C. C. et al. Distinct angiogenesis roles and surface markers of early and late endothelial progenitor cells revealed by functional group analyses. BMC Genom. 14, 182 (2013).

15. Liao, K. H. et al. Endothelial angiogenesis is directed by RUNX1T1-regulated VEGFA, BMP4 and TGF-beta2 expression. PLoS One 12, e0179758 (2017).

16. Alfayez, M., Vishnubalaji, R. \& Alajez, N. M. Runt-related transcription factor 1 (RUNX1T1) suppresses colorectal cancer cells through regulation of cell proliferation and chemotherapeutic drug resistance. Anticancer Res 36, 5257-5263 (2016).

17. Bashanfer, S. A. A., Saleem, M., Heidenreich, O., Moses, E. J. \& Yusoff, N. M. Disruption of MAPK1 expression in the ERK signalling pathway and the RUNX1RUNX1T1 fusion gene attenuate the differentiation and proliferation and induces the growth arrest in $\mathrm{t}(8 ; 21)$ leukaemia cells. Oncol. Rep. 41, 2027-2040 (2019).

18. Baby, N., Li, Y., Ling, E. A., Lu, J. \& Dheen, S. T. Runx1t1 (Runt-related transcription factor 1 ; translocated to, 1) epigenetically regulates the proliferation and nitric oxide production of microglia. PLoS One 9, e89326 (2014).
19. Liu, Y. et al. Upregulation of ERp57 promotes clear cell renal cell carcinoma progression by initiating a STAT3/LLF3 feedback loop. J. Exp. Clin. Cancer Res. 38, 439 (2019).

20. Yang, Z. et al. Silencing of miR-193a-5p increases the chemosensitivity of prostate cancer cells to docetaxel. J. Exp. Clin. Cancer Res. 36, 178 (2017).

21. Bayly, R. et al. E2A-PBX1 interacts directly with the KIX domain of CBP/p300 in the induction of proliferation in primary hematopoietic cells. J. Biol. Chem. 279, 55362-55371 (2004).

22. Yang, Z. et al. Dysregulation of p53-RBM25-mediated circAMOTL1L biogenesis contributes to prostate cancer progression through the circAMOTL1L-miR193a-5p-Pcdha pathway. Oncogene 38, 2516-2532 (2019).

23. Lewis, B. P., Burge, C. B. \& Bartel, D. P. Conserved seed pairing, often flanked by adenosines, indicates that thousands of human genes are microRNA targets. Cell 120, 15-20 (2005).

24. Betel, D., Koppal, A., Agius, P., Sander, C. \& Leslie, C. Comprehensive modeling of microRNA targets predicts functional non-conserved and non-canonical sites. Genome Biol. 11, R90 (2010).

25. Liu, J., Kong, X., Zhang, M., Yang, X. \& Xu, X. RNA binding protein 24 deletion disrupts global alternative splicing and causes dilated cardiomyopathy. Protein Cell 10, 405-416 (2019).

26. Rossetti, S., Hoogeveen, A. T. \& Sacchi, N. The MTG proteins: chromatin repression players with a passion for networking. Genomics 84, 1-9 (2004).

27. Davis, J. N., McGhee, L. \& Meyers, S. The ETO (MTG8) gene family. Gene $\mathbf{3 0 3}$, 1-10 (2003).

28. Oliveira, C., Faoro, H., Alves, L. R. \& Goldenberg, S. RNA-binding proteins and their role in the regulation of gene expression in Trypanosoma cruzi and Saccharomyces cerevisiae. Genet Mol. Biol. 40, 22-30 (2017).

29. Glisovic, T., Bachorik, J. L., Yong, J. \& Dreyfuss, G. RNA-binding proteins and post-transcriptional gene regulation. FEBS Lett. 582, 1977-1986 (2008).

30. Zhang, T. et al. Rbm24 regulates alternative splicing switch in embryonic stem cell cardiac lineage differentiation. Stem Cells 34, 1776-1789 (2016).

31. Mukherjee, N. et al. Deciphering human ribonucleoprotein regulatory networks. Nucleic Acids Res. 47, 570-581 (2019).

32. Khund-Sayeed, S. et al. 5-Hydroxymethylcytosine in E-box motifs ACAT|GTG and ACAC|GTG increases DNA-binding of the B-HLH transcription factor TCF4. Integr. Biol. 8, 936-945 (2016).

33. Patel, J. et al. Inhibition of C-terminal binding protein attenuates transcription factor 4 signaling to selectively target colon cancer stem cells. Cell Cycle 13, 3506-3518 (2014).

34. Ruiz de Garibay, G., et al. Tumor xenograft modeling identifies an association between TCF4 loss and breast cancer chemoresistance. Dis. Model. Mech 11,032292 (2018).

35. in 't Hout, F. E., van der Reijden, B. A., Monteferrario, D., Jansen, J. H. \& Huls, G. High expression of transcription factor 4 (TCF4) is an independent adverse prognostic factor in acute myeloid leukemia that could guide treatment decisions. Haematologica 99, e257-e259 (2014). 